\title{
ANÁLISE PARA VIABILIDADE ECONÔMICA DE INVESTIMENTO EM INDÚSTRIA DE INJEÇÃO DE MATERIAL PLÁSTICO UTILIZANDO MÉTODO DE ANÁLISE DE MONTE CARLO (AMC)
}

\section{MONTE CARLO METHOD (MCM) ANALYSES APPLIED TO THE DETERMINATION OF THE INVESTMENT IN A INJECTION PLASTIC INDUSTRY}

Pedro Paulo D’Alkmin Macedo, Rafael Maik de Oliveira, Juliano Augusto Nunes Paixão, Marcelo Pondé do Vale

Filiação ${ }^{1}$; Filiação ${ }^{2}$;

E-mail ${ }^{1} ; E^{2}-$ mail $^{2}$;

RESUMO - O mercado de itens personalizados para eventos como formaturas, casamentos estão em grande crescimento, sendo os copos em poliestireno um dos mais vendidos pela sua originalidade. Baseando-se neste cenário novos investidores estão surgindo neste mercado promissor, este trabalho visa elaborar uma análise de simulação de Monte Carlo para verificar a viabilidade de uma atividade de fabricação de copos utilizando uma injetora plástica. Entretanto, diante do alto custo envolvido, torna-se necessário um estudo para avaliar a viabilidade econômica da aquisição da máquina e suas instalações, de modo a determinar se o investimento está ao alcance do investidor e quais seriam os lucros adicionais advindos dessa aquisição. Com este objetivo desenvolveu-se um modelo que permitiu simular a distribuição de probabilidades do comportamento de cada uma das variáveis de previsão, Taxa interna de retorno (TIR), Valor presente líquido(VPL). Baseado neste projetou-se os fluxos de caixas futuros que permitiram completar a analise quanto à possibilidade de desenvolvimento da produção da empresa. Os resultados obtidos demonstram que o empreendedor obterá retorno financeiro com este investimento porque novas oportunidades de negócio serão criadas pelo aumento na demanda, pelo aumento na gama de produtos ofertados ao mercado de eventos ou mesmo com a prestação de serviço a terceiros.

Palavras-chave: Método de Monte Carlo; Indústria de injeção de material plástico; Viabilidade econômica; TIR; VPL

ABSTRACT - The Market for personalized items at events like weddings and graduation parties are expanding nowadays and one of the most sold items is the polystyrene glasses because its originality. Based on this scenario new investors are attempting to this promising market. This article attempts to make a simulated Monte Carlo analysis of 
polystyrene glasses production based on a plastic injection molding machine, a Himaco $120 \mathrm{H}$. Otherwise, this analysis is important and extremely necessary because of the high costs involved in the machine acquisition and in its installation to determinate if the investment is profitable for the investor. Considering this objective were developed a mathematical model that allowed to simulate the possible behaviors for each forecast variable like Internal Rate of Return - IRR and Net Present Value - NPV. Considering this, future cash flows were projected intending to complete the analyses for the company production development. The analyses results showed that the entrepreneur will reach financial return with this investment because new business opportunities will be created by increased demand, by the increase in the number of products offered to the event market or even by service delivered to other companies.

Keywords: Monte Carlo Method; injection plastic industry; economic viability; IRR; NPV

\section{INTRODUÇÃO}

É certo que o processo de geração e avaliação de alternativas de investimento é uma das questões mais importantes no desenvolvimento empresarial. Isto porque, através da análise de um projeto de investimento, se torna possível considerar os riscos decorrentes e também, os ajustes necessários para viabilização dos custos de produção sob pena se aceitar ou rejeitar projetos inadequados para o negócio da empresa como um todo. E assim sendo, há de se buscar, necessariamente, uma decisão qualificada quando se trata de investimentos para não se perder dinheiro ou colocar em risco a credibilidade da empresa.

Com o aquecimento do mercado de brindes personalizados para distribuição em eventos como formaturas casamentos e festas estudantis, a procura por copos em poliestireno se mostra em franco crescimento como um dos itens mais vendidos pela sua originalidade e baixo custo unitário.

$\mathrm{E}$, baseando-se nesse cenário e mercado promissor, o presente trabalho vai testar a aplicabilidade do Método de Simulação de Monte Carlo (SMC), como ferramenta a ser utilizada pelos novos investidores no ambiente empresarial para análise de riscos e verificar a viabilidade da fabricação de copos plásticos utilizando uma injetora modelo HIMACO $120 \mathrm{H}$, a ser adquirida.

Este método trata-se de uma simulação através de sistemas computacionais para o desenvolvimento de um modelo ou representação de uma situação real ou almejada, ou seja, uma ferramenta de auxílio na avaliação de resultados em vários cenários, que possibilitará uma melhor compreensão 
dentro do processo decisório sobre novos investimentos.

Nesse contexto, será uma análise de risco utilizando o método escolhido para apurar a necessidade ou não do investimento (compra de máquina injetora plástica), no processo de fabricação de copos plásticos para suprir a alta demanda.

Com esse intuito, foi construído um modelo de simulação com utilização de métodos matemáticos para gerar a distribuição de probabilidades do comportamento de cada uma das variáveis de previsão, Taxa Interna de Retorno (TIR), Valor Presente Líquido (VPL). Também, foram projetados os fluxos de caixas futuros para analisar o problema.

Este trabalho está organizado da seguinte forma: apresenta uma introdução que traça os objetivos gerais da pesquisa e método escolhido (Simulação Monte Carlo); uma revisão bibliográfica do método escolhido (SMC); a demonstração da empresa analisada; a descrição do processo produtivo onde são detalhadas as etapas produtivas e formuladas as novas previsões através da simulação.

Como conclusão, faz se uma síntese dos resultados após as execuções da programação e análise da aplicabilidade da simulação (método de Monte Carlo) como ferramenta eficiente para auxílio dos gestores e empresários diante de processo decisório para novos investimentos.

\section{REVISÃO DE LITERATURA}

O objetivo deste trabalho é testar a aplicabilidade do método de Simulação Monte Carlo (SMC) na previsão de variações nos custos de produção, análises de riscos e retorno de investimentos quando da aquisição de uma nova máquina injetora modelo HIMACO $120 \mathrm{H}$ para produção de copos plásticos.

\section{Análise de riscos}

Através das projeções do fluxo de caixa se faz possível a análise de riscos, com a determinação dos custos de capital e a viabilização da utilização de técnicas determinísticas e probabilísticas.

Isto porque, a partir de técnicas de avaliação são determinados os benefícios futuros derivados da aceitação do investimento, e também, se pode obter informações que possibilitam ou não o investimento, sendo fruto de um modelo determinístico e probabilístico.

$$
\text { Para ABREU (1982, p. 96), “ } a
$$
medição e análise de risco de projetos de investimentos possibilitam conhecer os resultados consequentes de cada cenário possivel, e a probabilidade de ocorrência de cada um deles. Conquanto, a análise de investimentos se considerando os fatores de 
risco pode ser desenvolvida através das técnicas de análise de cenários, análise de sensibilidade e simulação de Monte Carlo".

\section{Modelos probabilísticos}

A utilização de métodos probabilísticos para avaliação de projetos de investimentos é aplicada sempre a partir de um fator de incerteza, ou seja, através da aleatoriedade das variáveis que compõem o modelo a ser pesquisado e confrontado. Nesse sentido, ensina CORREIA NETO (2009, p. 4) que a "incerteza é uma característica comum aos projetos, uma vez que todo ato de projeção envolve algum nível de incerteza".

Dessa forma, o fato de não se saber se as projeções acontecerão conforme as previsões, por si só, já agrega um nível de incerteza aa todo projeto de investimento. É isso que se verá a seguir.

\section{Simulação de Monte Carlo - SMC}

A simulação é uma ferramenta de análise quantitativa que pode ser utilizada na geração de cenários futuros e situações alternativas antes de mudanças ou novos investimentos dentro da cadeia produtiva.

Atualmente, são conhecidos dois modelos de simulação: o determinístico e o probabilístico. No modelo determinístico, os dados são obtidos com certeza e não se leva em conta, as probabilidades de que o valor escolhido possa sofrer alterações no futuro. Já, o modelo probabilístico incorpora todos os comportamentos prováveis dentro do sistema, na tentativa de capturar as variáveis que cercam o sistema por meio de métodos estatísticos e de computadores.

O modelo probabilístico, como já visto, teve sua origem no método de Simulação de Monte Carlo através de simulações aleatórias, introduzindo-se a análise de riscos, a variáveis ambientais e os elementos de incerteza implícitos.

Para HAMMERSLEY e HANDSCOMB (1964, p. 3), o Método de Simulação de Monte Carlo, pode ser definido como sendo "a parte da matemática experimental que está preocupada em experiências com números aleatórios".

Os números são obtidos de artifícios aleatórios (por exemplo: tabelas, roletas, sorteios) ou diretamente de softwares, através de funções específicas. E, a cada iteração, o resultado é armazenado para ao final de todas as repetições (sequência de resultados gerados) sejam transformadas numa distribuição de frequência que possibilita calcular estatísticas descritivas como valor esperado (média), valor mínimo, valor máximo e desvio padrão.

Por isso, esse tipo de simulação é aplicado em problemas de tomada de decisão que envolva riscos e incerteza, portanto, situações nas quais o 
comportamento das variáveis envolvidas com o problema é de natureza não determinística.

Para uma correta operacionalização do método, LUSTOSA, PONTE e DOMINAS (op. cit, p. 252) explicam "que a simulação deve ser replicada mais de cem vezes para que se obtenha uma amostra representativa". Mas, não há recomendação quanto ao número máximo de simulações a serem realizadas em cada pesquisa.

\section{Elaboração do modelo}

Ao ser utilizado a simulação de Monte Carlo se faz necessário a elaboração de um modelo no qual represente a situação podendo ser executada para simulação de cenários futuros.

A entrada de uma variável estocástica no sistema se dá por meio de sua associação a uma distribuição teórica de probabilidades, e para que isso ocorra, a série de valores possíveis é submetida a um teste de aderência. E, uma vez inseridas todas as variáveis determinísticas e estocásticas, a construção do modelo é finalizada com a definição das relações entre elas, que correspondem aos cálculos necessários para a determinação (saída) das variáveis de previsão.

\section{Execução do modelo}

Foi construído um modelo para execução em looping composto por duas etapas. Na primeira etapa, o simulador instalado no sistema computadorizado atribuirá para cada variável estocástica, um valor aleatório da distribuição associado à variável e, na segunda etapa, os valores gerados de cada variável estocástica serão combinados com os valores determinísticos para cálculo da variável de previsão. Assim, a cada execução do looping, um fluxo de caixa é gerado e a partir deste fluxo, então, será possível calcular os valores de VPL (valor presente líquido) e da TIR (taxa interna de retorno). E, com a execução de um determinado número (n) de loopings, é possível construir uma distribuição de probabilidade para cada variável de previsão, isso porque o sistema computadorizado permite a execução mais de mil loopings em segundos.

\section{Resultados apurados na análise}

Dessa forma, na análise de risco se tem o valor esperado do valor presente líquido - VPL, representando a média dos VPLs gerados pelo modelo.

CORREIA NETO (op. cit., p. 199) atenta para "o fato de que o retorno do projeto é esperado, uma vez que são calculados sobre valores projetados, ainda não realizados e passíveis de desvios. Assim como, a média da distribuição dá o valor esperado, o desvio padrão mede a dispersão em torno dessa média". 
E, essa característica mutável das variáveis estocásticas pode levar a situações favoráveis ou desfavoráveis à viabilidade do projeto, quando se aumenta ou diminui os valores indicados como VPL e TIR. Também, a análise da dispersão é importante, quando o desvio padrão se mostrar alto, podem ocorrer situações que apresentam um retorno modesto e noutras, onde o retorno é significativo.

Portanto, é de suma importância a análise do desvio padrão que retrata o risco associado ao investimento no projeto estudado. Se o desvio padrão é alto, menor é a probabilidade de ocorrência do valor esperado e maior, a probabilidade da ocorrência de valores distantes da média.

\section{METODOLOGIA DE ESTUDO}

\section{Descrição da empresa}

Trata-se de empresa que fabrica e comercializa copos plásticos personalizados de poliestireno chamados de long drinks. No processo de produção dos copos é utilizado sistema de injeção plástica.

\section{Descrição do processo}

O processo de produção dos copos foi sintetizado nas etapas abaixo:

1. Avanço da placa móvel e fechamento do molde;

2. Injeção: período em que a unidade de injeção permanece na posição avançada durante um tempo determinado em função da natureza do material, do tamanho e da forma do produto a ser moldado;

3. Recalque: onde é feita a compactação do material plástico para compensação da contração do mesmo no interior da cavidade do molde;

4. Dosagem do material, na quantidade a ser injetada;

5. Descompressão da unidade injetora;

6. Tempo de resfriamento, variando de acordo com a espessura da parede e tamanho da peça;

7. Abertura do molde e avanço do extrator para retirar a peça do molde;

8. Se estiver programada no modo automático, após a extração da peça, a injetora iniciará um novo ciclo. Mas, se estiver no modo semiautomático, variando de acordo com modelo da máquina, a porta deverá ser aberta ou fechada novamente ou um botão acionado para reiniciar outro ciclo. 


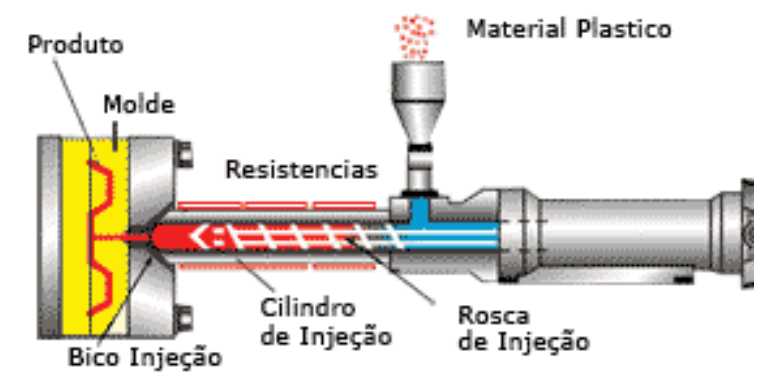

Figura 1. Injetora em funcionamento

(Fonte: eletroplasticoscaramuru.com.br)

\section{Construção do modelo}

Será desenvolvida uma pesquisa de campo para levantamento dos custos, receitas e o incremental do investimento (nova máquina injetora) em 05 (cinco) períodos para que seja estabelecido um fluxo de caixa retratando a utilização do ativo a ser adquirido.

De acordo com modelo do fluxo, as variáveis são classificadas de acordo com sua relevância e aderência para com o modelo pesquisado (ou seja, valor absoluto atribuído ou distribuição de probabilidade) numa planilha do $\operatorname{Excel}^{\circledR}$, através da utilização da manipulação dos dados do suplemento Visual Basic Aplication.

\section{Projeção do investimento inicial}

Os investimentos iniciais totalizam o valor de $\mathrm{R} \$ 7.700,00$ (sete mil e setecentos reais), e serão constituídos por: 1 (uma) máquina injetora $\mathrm{MG}, 80$ toneladas, no valor de $\mathrm{R} \$ 5.000,00$ (cinco mil reais);1 (uma) torre de resfriamento, Marca Kurper, no valor de
R\$ 1.000,00 (um mil reais); infraestrutura (tubulações, mangueiras, padrão de energia, etc.) no valo de $\mathrm{R} \$ 1.500,00$ (um mil e quinhentos reais): instalações e adequações para funcionamento do maquinário no valor de $\mathrm{R} \$ 200,00$ (duzentos reais).

\section{Projeção dos fluxos de caixa}

Ao se adquirir umas das máquinas acarretarão variações nos custos da empresa de acordo com a opção do investimento.

\section{a) Custo da matéria prima: $\mathrm{A}$} composição do produto é apresentada na figura 2. abaixo e os custos podem variar de acordo com a quantidade comprada. O custo unitário de matéria prima foi determinado de acordo com a percentagem de material utilizado na composição multiplicado pelo custo do quilograma ( $\mathrm{kg})$, e também, pelo peso respectivo da peça, e somado ao valor da embalagem (obtido quando dividido o valor do pacote pelo número de unidades). Foi então, estabelecida a distribuição 


\begin{tabular}{|c|c|c|c|c|c|}
\hline Peso da peça & 0,050 & Determinístico & & & $\mathrm{kg}$ \\
\hline Pigmento por peça & $2 \%$ & Determinístico & & & $\%$ \\
\hline Material granulado/peça & $98 \%$ & Determinístico & & & $\%$ \\
\hline Preço do material granulado & $\mathrm{R} \$ \mathbf{T} \quad 7,40$ & Triangular & $\mathrm{R} \$ \mathbf{7} \quad 7,00$ & $\mathrm{R} \$ \mathbf{1 2}, 00$ & $\mathrm{R} \$ / \mathrm{kg}$ \\
\hline Preço do pigmento & $\mathrm{R} \$ \quad 20,00$ & Triangular & $\mathrm{R} \$ 18,00$ & $\mathrm{R} \$ \quad 30,00$ & $\mathrm{R} \$ \mathbf{k g}$ \\
\hline Embalagem & $\begin{array}{r}\mathrm{R} \$ \quad 8,50 \\
\end{array}$ & Triangular & $\mathrm{R} \$ \quad 7,00$ & $R \$ \quad 10,50$ & 500 embalagem \\
\hline
\end{tabular}

Figura 2. Demonstração dos custos e composição do produto

Fonte: elaborado pelos autores

b) Mão de obra direta: o próprio empresário desempenhará a atividade inicial com os custos previdenciários orçados dentro da alíquota de 5,96\% através do plano estatal do SUPER SIMPLES. c) Custos de fabricação e manutenção dos moldes: os custos de manutenção do molde e da máquina serão atribuídos de acordo com uma percentagem de seu valor e podendo variar com o tempo e forma de utilização através da distribuição triangular.

\begin{tabular}{|l|c|l|l|l|l|}
\hline Manutenção molde & $2 \%$ & Triangular & $1 \%$ & $6 \%$ & Valor inicial da maq \\
\hline Manutenção máquina & $2 \%$ & Triangular & $1 \%$ & $6 \%$ & Valor inicial da maq \\
\hline
\end{tabular}

Figura 3. Atribuição dos custos e variação do parâmetro dentro da análise.

Fonte: Elaborado pelos autores

d) Energia elétrica: 0 custo de energia elétrica de acordo com a utilização da injetora e torre de resfriamento foi determinado e atribuído uma distribuição triangular visando estabelecer possível variação na tarifa.

\begin{tabular}{|c|c|c|c|c|c|c|c|c|}
\hline Energia elétrica & $\mathrm{RS}$ & 450,00 & Triangular & $\mathrm{R} \$$ & 400,00 & $\mathrm{RS}$ & 750,00 & Mês \\
\hline
\end{tabular}

Figura 4. Tarifa aplicada e as variações do parâmetro

Fonte: elaborado pelos autores

e) Demanda, preço e volume de venda, e receita: as possíveis variações na demanda e nas vendas foram projetadas visando atribuir diferentes cenários para 0 desenvolvimento da atividade, e também, o preço foi projetado de acordo com a variação da margem do produto. As receitas dos períodos pesquisados são obtidas através da multiplicação da venda mensal pelo valor unitário. 


\begin{tabular}{|l|c|l|l|l|l|}
\hline Produtividade mensal & 19008 & Triangular & 14000 & 20064 & unidade \\
\hline Demanda & 15000 & Triangular & 6000 & 20064 & $\mathrm{R} \$$ \\
\hline Venda mensal & 15000 & Triangular & 6000 & 20064 & $\mathrm{R} \$$ \\
\hline Preço unitário & 1,1 & Triangular & 0,85 & 1,2 & $\mathrm{R} \$$ \\
\hline
\end{tabular}

Figura 5. Variação de demanda, volume de venda e demanda Fonte: elaborado pelos autores

taxa de utilização, o produto entre o tempo e

f) Produtividade: foi estabelecida de a taxa de utilização, multiplicando a acordo com a capacidade da máquina, o capacidade se obteve produtividade mensal. tempo trabalhado em relação á um mês e a

\begin{tabular}{|l|c|l|l|l|l|}
\hline Dias trabalhados & 22 & Determinístico & 22 & 22 & Dias \\
\hline Produtividade & 2 & Determinístico & & & pç/min \\
\hline Taxa de utilização & $90 \%$ & Triangular & $70 \%$ & $95 \%$ & tempo \\
\hline
\end{tabular}

Figura 6. Valores atribuídos ao modelo

Fonte: elaborado pelos autores

g) Valor residual e depreciação: o bem foi depreciado linearmente em 05 (cinco) anos e ao fim do período, foi the atribuído um valor residual médio efetuando considerações das variações possíveis no fim da atividade.

\section{Metodologia de cálculo dos fluxos de caixa projetados}

É possível a obtenção da receita bruta mensal através do produto da venda mensal pelo valor unitário comercializado, e subtraindo os impostos sobre a receita, para determinação da receita líquida do período. Somam-se os custos fixos constituídos de tarifa de energia, custos de manutenção, custos variáveis (compra de poliestireno, pigmento, embalagem, etc.) que subtraído da receita líquida, para obtenção do valor do LAJIDA (lucro antes de juros, impostos, depreciação e amortização).

Como no modelo analisado não há financiamento, o valor do LAJIDA será o mesmo do LAIR (lucro antes do imposto de renda). Então, a partir do LAIR, deve ser subtraída a depreciação e o Imposto de Renda (com alíquota de 30\%) para caracterização do lucro contábil do período. Posteriormente, se somará a depreciação para obtenção do fluxo operacional mensal.

\section{Cálculo do VPL e TIR}

Com os valores do fluxo operacional mensal, acima explicado, multiplicados pelo 
número de meses analisados, obtivemos o fluxo anual do investimento aliado à taxa de atratividade para análise dos indicadores, TIR e VPL, do modelo durante 05 (cinco) períodos.

\section{Execução do modelo}

Determinando a quantidade de simulações a serem efetuadas pelo programa, os valores das variáveis estocásticas (variáveis aleatórias) são combinados as determinísticas. Executando o programa é possível obter o saldo da atividade de forma mensal conforme a distribuição atribuída a cada variável, através da variação do indicador TIR.

\section{ANÁLISE DE SENSIBILIDADE DO MODELO}

A análise de sensibilidade foi elaborada em conjunto com a simulação de Monte Carlo com um objetivo importante, o estabelecimento das variáveis mais impactantes dentro dos indicadores da atividade e que se refletem através da TIR dentro de faixas possíveis no decorrer do tempo. E, visando sempre, encontrar a variável com maior influência no sucesso do modelo, foram efetuadas as simulações com identificação do volume de vendas.

Nessa análise foram observados os seguintes parâmetros: a) 6.500 unidades/mês com um passo de 100 unidades até o valor de 8.000 unidades, o que facilitou a identificação a partir do ponto onde a atividade atingiria, economicamente, um resultado satisfatório; e b) um desvio padrão de $79 \%$ (setenta e nove por cento).

É importante ressaltar que, há uma limitação no valor máximo da quantidade de venda para uma melhor análise do ponto crítico do projeto, ou seja, acima de 8.000 unidades vendidas, a TIR tende ser positiva e crescente.

\section{ANÁLISE DE RESULTADOS}

O modelo foi submetido a 10.000 (dez mil) simulações para observação do comportamento dos indicadores e foi estabelecida uma TIR média de 949,00\% (novecentos e quarenta e nove por cento) durante os 204s (duzentos e quatro segundos) de execução em se utilizando um computador Positivo SIM 6240, com Processador Intel Core 13, Memória RAM de 4GB.

Com as simulações foram obtidos os valores de TIR mínima de 121,66\% e máxima de 1.940,31\%, e, apresentado um desvio padrão de 300,34\%. Também, não houve considerações de insucessos.

É de se concluir que, que o modelo de injetora analisado trata-se de alternativa extremamente atraente, dado aos baixos custos de investimento e operação e, aliados a uma boa margem de contribuição. E, a apurado o alto nível de sucesso do investimento analisado, a próxima análise 
seria buscar uma alternativa onde a empresa pudesse investir um maior capital em equipamentos para eliminação do gargalo na capacidade produtiva.

A opção encontrada foi o investimento numa máquina injetora, modelo HIMACO 120H, avaliada em $\mathrm{R} \$$ $45.000,00$ (quarenta e cinco mil reais) e numa ferramenta de duas cavidades a ser acoplada à injetora avaliada em $\mathrm{R} \$ 26.000,00$ (vinte e seis mil reais) e com isso, a capacidade produtiva seria aumentada substancialmente (produção de 02 copos para 05 copos por minuto).

O mesmo modelo de análise foi utilizado (modificação das variáveis) para as novas simulações e, após as 10.000 (dez mil) simulações, com tempo de execução de 166s (cento e sessenta e seis segundos), ficou demonstrada uma TIR média de 120,39\%, com índices mínimos de $-31,38 \%$ e máximo de $333,60 \%$. O desvio padrão apresentado foi de $55,83 \%$ e os insucessos na ordem de $0,66 \%$, quando a TIR apresentou valores negativos.

Ao final restou demonstrada ser esta opção, um investimento satisfatório porque pode agregar outras atividades, ou seja, a sua capacidade produtiva aumentaria de acordo com a atratividade de seus indicadores econômicos.

\section{CONCLUSÃO}

A realização da análise do investimento em cenários simulados e passíveis de serem confrontados ao longo do desenvolvimento da atividade produtiva tornou possível demonstrar que a aquisição da máquina injetora e os demais complementos seria um investimento com alta TIR (taxa interna de retorno), que se manteve com uma média de 949\%.

Também, foi realizada uma análise de sensibilidade para identificação do fator que provocasse maior impacto dentro do modelo projetado, e sendo constatado que o volume de vendas representava o maior risco, foram tomadas as medidas necessárias de controle dos riscos.

No tocante à segunda simulação desenvolvida, foi possível concluir que, caso não houvesse um aumento significativo na demanda, a máquina poderia ficar ociosa. Mas, essa ociosidade poderia ser contornada se aliada às novas alternativas, como aluguel ou desenvolvimento de outros produtos, que aumentariam a viabilidade do investimento e melhorariam os indicadores da atividade.

A partir dessas constatações, podese inferir que o objetivo proposto foi alcançado e que a Simulação de Monte Carlo mostrou-se um método apropriado para prever os valores (custos, TIR, VCL, etc.) em se adequando as variáveis simuladas aos impactos do fator modificativo (nova 
máquina injetora para produção de copos plásticos em maior escala).

No caso em tela, como a empresa de copos plásticos está iniciando suas atividades, realização dessa análise (com variações e simulações) foi de grande valia, porque viabilizou a aquisição da máquina Injetora HIMACO 120H, como um investimento altamente satisfatório, e também, a possibilidade de ampliação e diversificação da sua linha de produtos.

Assim sendo, é possível afirmar que o método de Simulação de Monte Carlo se apresenta como ferramenta eficaz no auxílio à visualização de diversas situações e variações, inclusive em cenários contrastantes, nos processos decisórios para investimentos futuros.

\section{REFERÊNCIAS}

ABREU, Paulo Fernando Simas Peixoto de; STEPHAN, Christian. Análise de Investimentos. Rio de Janeiro: Campus, 1982.

ANDRADE, E. L. Introdução à Pesquisa Operacional - Métodos e Modelos para a Análise de Decisão. Rio de Janeiro: LTC Livros Técnicos e Científicos Ed., 1989.

BARROS, N. R; LUSTOSA, P. R. B e GARCIA, Solange. Aplicabilidade do Método de Simulação de Monte Carlo na Previsão dos Custos de Produção de Companhias Industriais: O Caso da Companhia Vale do Rio Doce. In: RCO - Revista de Contabilidade e Organizações - FEA-RP/USP, v. 4, n. 10, p. 173-173, set-dez 2010.
CORREIA NETO, Jocildo Figueredo. Elaboração e avaliação de projetos de investimento: considerando o risco. Rio de Janeiro: Elsevier, 2009.

ESCUDERO, L. F. La simulación en la empresa. Barraincúa: Deusto, 1973.

HAMMERSLEY, J.M e HANDSCOMB, D. C. Monte Carlo methods. London: Methuen,1964. http://dx.doi.org/10.1007/978-94-009-5819$\underline{7}$

LUSTOSA, P. R. B.; PONTE, V. M. R.; DOMINAS, W. R. Simulação. In: CORRAR, L. J.; THEÒPHILO, C. R. (Orgs.). Pesquisa operacional para decisão em Contabilidade e Administração. São Paulo: Atlas, 2004.

NASCIMENTO, A. M.; ZUCCHI, A. L. Modelos de simulação. São Paulo, Universidade de São Paulo, 1997. Monografia - Faculdade de Economia, Administração e Contabilidade, Universidade de São Paulo, São Paulo, 1997.

SARAIVA JÚNIOR, A. F. et al. Simulação de Monte Carlo aplicada à análise econômica de pedido. Produção, v. 21, n. 1, p. 149-164, jan./mar. 2011.

http://dx.doi.org/10.1590/S010365132011005000016

STEVENSON, William J. Estatística aplicada à Administração. São Paulo: Harper \& Row do Brasil, 1981. 